\title{
Assessing the quality of life of patients with intestinal failure on home parenteral nutrition
}

\author{
D M Richards, M H Irving
}

\begin{abstract}
Background-A study was performed to measure the quality of life of patients receiving home parenteral nutrition (HPN). Quality of life is an important determinant of the efffectiveness of health technologies, but it has rarely been assessed in patients receiving HPN.

Aims-To measure quality of life and highlight any moderating factors.

Patients and methods-Quality of life was measured using two validated instruments (SF 36 and EuroQol) in 51 patients with intestinal failure. All patients had benign disease, the commonest being Crohn's disease $(\mathbf{n}=35)$.

Results-HPN patient scores were worse for six of eight SF 36 domains $(p<0.05)$ compared with standard population scores. Younger patients $(<45)$ had good scores but older patients ( $>55$ years) scored significantly less. Patients addicted to narcotic substances had very low scores. EuroQol utility scores confirmed the SF results. Forty one patients reported that they felt too ill to work and only five were in full time work or education.

Conclusions-The health status profile of our young patients on HPN was good compared with the normal population. The poorest scores were in older patients and those dependent on narcotic drugs. This has clinical and economic relevance when considering such patients for HPN. (Gut 1997; 40: 218-222)
\end{abstract}

Keywords: intestinal failure, home parenteral nutrition, quality of life.

Home parenteral nutrition (HPN) is a highly technical, complex procedure that puts great demands on the patient and inevitably has an impact on quality of life. For patients with complete intestinal failure, $\mathrm{HPN}$ is lifesaving. Intestinal failure is defined as inadequate intestinal function for absorbtion of nutrients and electrolytes. Without the intravenous infusion of nutrients and electrolytes there is an almost inevitable progression from metabolic disturbance and malnutrition to death. ${ }^{1}$ At the present time the alternatives to HPN are prolonged hospital care or multiple hospital admissions for intravenous nutrition. In the long term, small bowel transplantation may be an answer to the problem of intestinal failure. Patients with intestinal failure can be dependent on total parenteral nutrition (TPN) for many years and one of the main aims of treatment must be to give the recipient the "best possible" quality of life. Assessments of quality of life using validated techniques have rarely been performed on this population of patients in the past. ${ }^{2}{ }^{3}$ Information on outcomes is one of the central facets of the United Kingdom Health Technology Assessment Programme and this study was performed to measure one of the outcomes of HPN.

The aim of this study was to describe a profile of health status of patients on the HPN programme, employing commonly used, well validated, subjective health status instruments and to examine which factors moderated outcomes.

\section{Methods}

\section{Patients}

The patients studied were monitored by the University of Manchester intestinal failure unit, based at Hope Hospital, Salford. At the time of the study, 64 patients were receiving TPN at home. The average age was $44 \cdot 4$ (range 17-70) years; $40(62 \cdot 5 \%)$ were women. All patients had intestinal failure secondary to benign disease. The commonest underlying diagnosis was Crohn's disease $(n=35,55 \%)$ followed by mesenteric vessel occlusion $(n=11$, $17 \%)$, and volvulus $(n=8,12 \cdot 5 \%)$. The other patients had various diagnoses $(n=10,15 \cdot 5 \%)$. All had received at least six months of nutrition at home. The median duration of HPN treatment was four (range 0.5-15) years. The HPN was infused on a cyclical nocturnal basis in 63 (98\%) patients for a median of six (range 2-7) nights. All patients were treated according to standard unit protocols. We estimate that we covered about $35 \%$ of the total United Kingdom population of patients receiving $\mathrm{HPN}^{4}$

\section{Methods}

A data extraction sheet was designed to collect demographic and clinical details from the case notes. We recorded age, sex, diagnosis and reason for intestinal failure, presence of an intestinal stoma, previous surgery, complications, duration of HPN, distance from home to the nutrition unit, and employment status.

The SF 36 health status questionnaire (United Kingdom version) were sent to each patient by post and was used to obtain a profile of health status. This instrument is designed to examine eight aspects of life (domains, Table I) and scores each domain on a scale of $0-100 \%$. The SF 36 instrument was chosen because the domains examined are particularly relevant to patients on HPN. EuroQol ${ }^{5}$ was used to obtain 
TABLE I The eight aspects of life examined with the SF 36 questionnaire

\begin{tabular}{ll}
\hline Concept & Summary of content \\
\hline Physical functioning (PF) & $\begin{array}{l}\text { Extent to which health limits physical activities such as self } \\
\text { care, walking, climbing stairs, bending, lifting, and moderate } \\
\text { to vigorous exercise. } \\
\text { Extent to which physical health interferes with work or other } \\
\text { daily activity. } \\
\text { Intensity of pain and effect of pain on normal work, both } \\
\text { inside and outside the home. }\end{array}$ \\
Role functioning-physical (RP) \\
Bersonal evaluation of health including current health, health \\
outlook, and resistance to illness.
\end{tabular}

TABLE II Mean SF 36 and EuroQol scores for patients on $H P N$ and significance of score $\mathrm{v}$ results from a large sample of the normal United States population

\begin{tabular}{lll}
\hline & Patients on HPN & Normal population \\
\hline Physical function & $45 \cdot 53$ & $84 \cdot 15^{\star}$ \\
Role-physical & 25 & $80 \cdot 96^{\star}$ \\
Body pain & $46 \cdot 12$ & $75 \cdot 15^{\star}$ \\
General health & $36 \cdot 33$ & $71 \cdot 95^{\star}$ \\
Vitality & $37 \cdot 87$ & $60 \cdot 86^{\star}$ \\
Social functioning & $50 \cdot 37$ & $83 \cdot 28^{\star}$ \\
Role-emotional & $58 \cdot 67$ & $81 \cdot 26$ (NS) \\
Mental health & $66 \cdot 68$ & $74 \cdot 74$ (NS) \\
\hline
\end{tabular}

^Significant difference between patients on HPN and the normal population.

TABLE III EuroQol results

\begin{tabular}{|c|c|}
\hline Index & Score \\
\hline $\begin{array}{l}\text { EuroQol index (mean } \\
\text { sample score) }\end{array}$ & $\begin{array}{l}0.51(0=\text { death; } 1=\text { best possible } \\
\text { quality of life })\end{array}$ \\
\hline $\begin{array}{l}\text { Self rated health } \\
\text { Mean population score }\end{array}$ & $\begin{array}{l}53 \cdot 3^{\star}(0=\text { death } ; 100=\text { best possible } \\
84 \text { quality of life })\end{array}$ \\
\hline
\end{tabular}

$\mathrm{p}=0 \cdot 05$; self rated health $v$ mean population score

utility scores which are equivalent to a single score for health status on a scale of zero to one $(0=$ death, $1=$ best possible quality of life). The domains covered by EuroQol are also relevant to the HPN population and include mobility, self care, activity, pain, and mental wellbeing. Self rated health status (scale $0=$ death, $100=$ best possible quality of life) and change in health status over the past 12 months are also recorded. Both questionnaires can be completed in less than 15 minutes, making them a suitable combination for postal distribution. Written reminders were sent to nonrespondents after four weeks.

The SF 36 questionnaires were scored according to the published scoring manual. ${ }^{6}$ EuroQol health states were valued according to the scoring sheet and self rated health states were compared with population "norms" (available from members of the EuroQol Group). We retested 10 patients $(16 \%)$ in the outpatient clinic to assess the reproducibility of the results. Before starting the study we calculated that 34 patients were required to make statistical inferences. ${ }^{7}$ The Mann-Witney $U$ test was used to determine significance.

\section{Results}

Fifty one (of 64) completed questionnaires were received. Forty one $(80 \%)$ patients reported that they were too ill to work. Only five $(10 \%)$ were in full or part time work or study and five $(10 \%)$ were able to cope with housework alone. Tables II and III show the mean SF 36 and EuroQol scores. The scores for physical function, physical role, emotional role, social function, vitality, and pain were all significantly lower than normal population scores (Table II). Emotional role and mental health scores were at the lower end of the normal range. No differences were seen between the patients who said that they were too ill to work and those who were in employment although small numbers in the employed group prevented meaningful analysis. The scores for the younger patients (age $<45$ ) were good (Fig 1), and approached those of the normal population in most of the domains tested. However, the scores for the older patients were poor and there were significantly

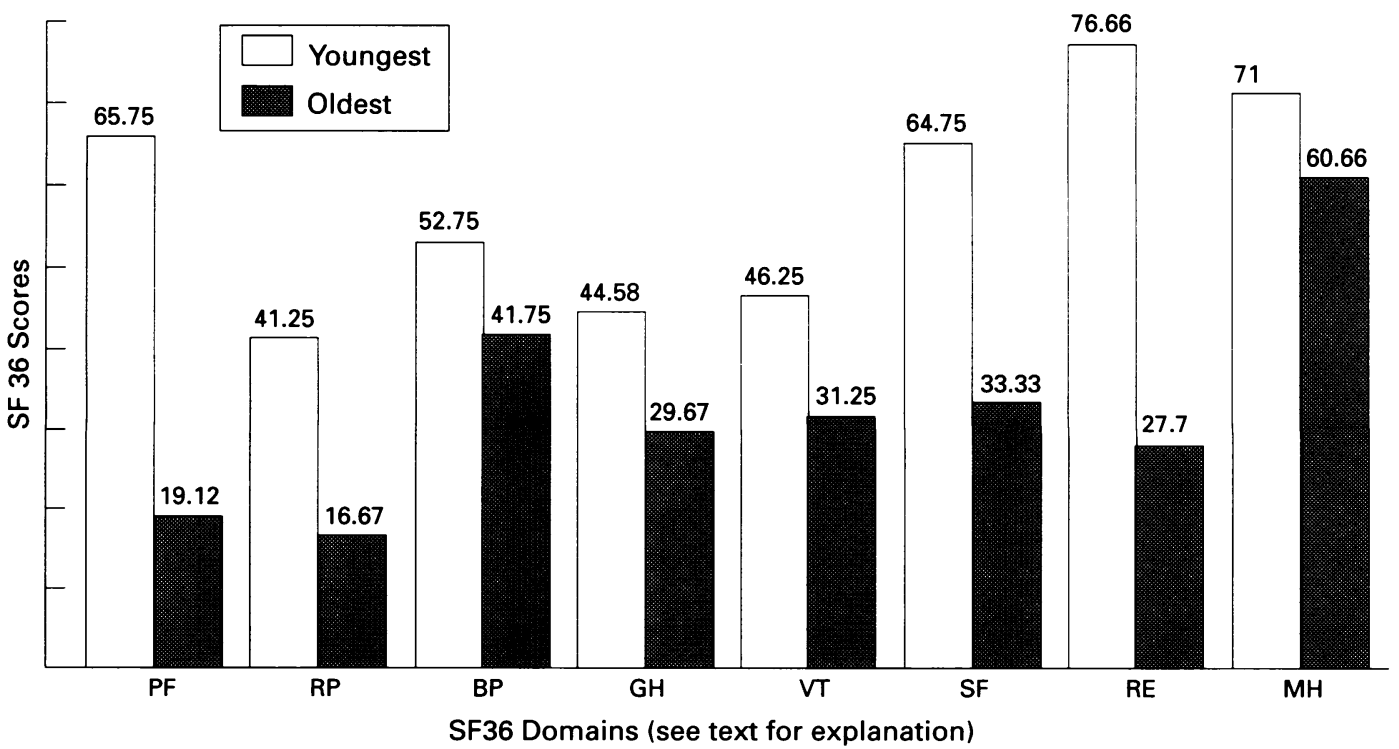

Figure 1: A comparison of SF 36 scores for younger and older patients. 


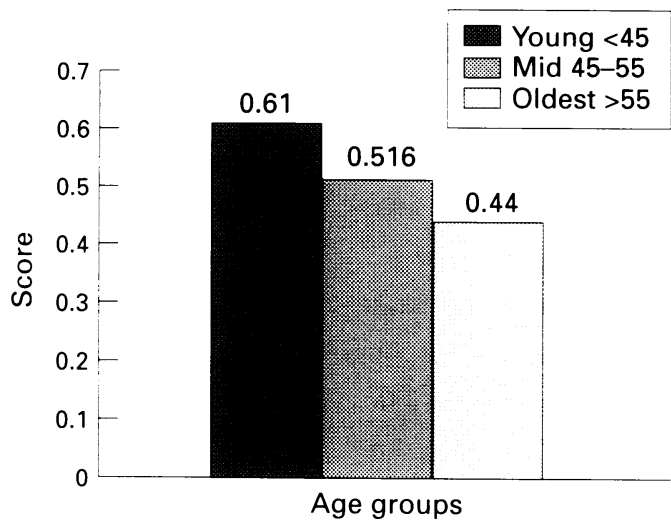

Figure 2: EuroQol scores for various age groups.

lower scores $(p<0.05)$ in the domains of physical functioning, social functioning, and emotional role (Fig 1). Scores for general health, physical role, and vitality approached significance $(p=0.08$ to 0.09$)$. Five patients who were addicted to narcotic analgesics had SF 36 domain scores which averaged $25 \%$, much lower than other patients receiving HPN. Twenty eight patients who had recently started HPN treatment ( $<3$ years) had similar scores to well established patients but the patients who had been on HPN longer had significantly better scores for social function $(\mathrm{p}<0.05)$. No differences were seen between the scores for men and women.

Table III shows the EuroQol index and self rated scores and Fig 2 shows the scores for the different age groups. The EuroQol scores showed very similar trends to the SF 36 results, and again a significant difference between the older and younger groups was apparent $(\mathrm{p}=0 \cdot 04)$.

No difference could be measured between those patients with or without a stoma or between the scores for patients who had had a complication requiring hospital admission and those who had not been in hospital. The scores for patients with Crohn's disease were similar to the scores for patients in other subgroups
(Fig 3) but the small numbers in the subgroups prevented firm conclusions. The significant trends of better scores for younger patients were also noted in the Crohn's disease group. The scores for patients with Crohn's disease receiving HPN were compared with scores for age matched patients with type II diabetes and congestive cardiac failure (Fig 4). Scores for the Crohn's disease group were lower in each of the domains examined.

Most patients remained in a constant health state, with $10 \%$ better and $10 \%$ worse off than one year previously.

The reproducibility checks showed a high correlation between the initial EuroQol scores and the retest scores $(0.96$ Pearson product moment correlation coefficient).

\section{Discussion}

Home parenteral nutrition was introduced in the United States in the late 1960s as a treatment for patients who had lost a significant degree of intestinal function and were unable to maintain an adequate nutritional state (intestinal failure). The effects of introducing this technology were that patients who had previously been dying of a combination of dehydration and malnutrition, survived and maintained a positive nitrogen balance.

The technology is complex and expensive and requires the patient to be dependent on a machine for nutritional support. The nature of the technology has an inevitable effect on quality of life. There have been some previous attempts to measure the quality of life of patients receiving HPN but rarely in the United Kingdom and usually with nonvalidated techniques. Occasionally, functional assessments have been performed, giving a crude objective indication of quality of life. ${ }^{8-10}$

Detsky et $a l^{2}$ compared quality of life scores for patients receiving HPN with scores for patients receiving all their nutrition in hospital. They found that moving patients from hospital to the home setting resulted in a significant gain in the quality of life score.

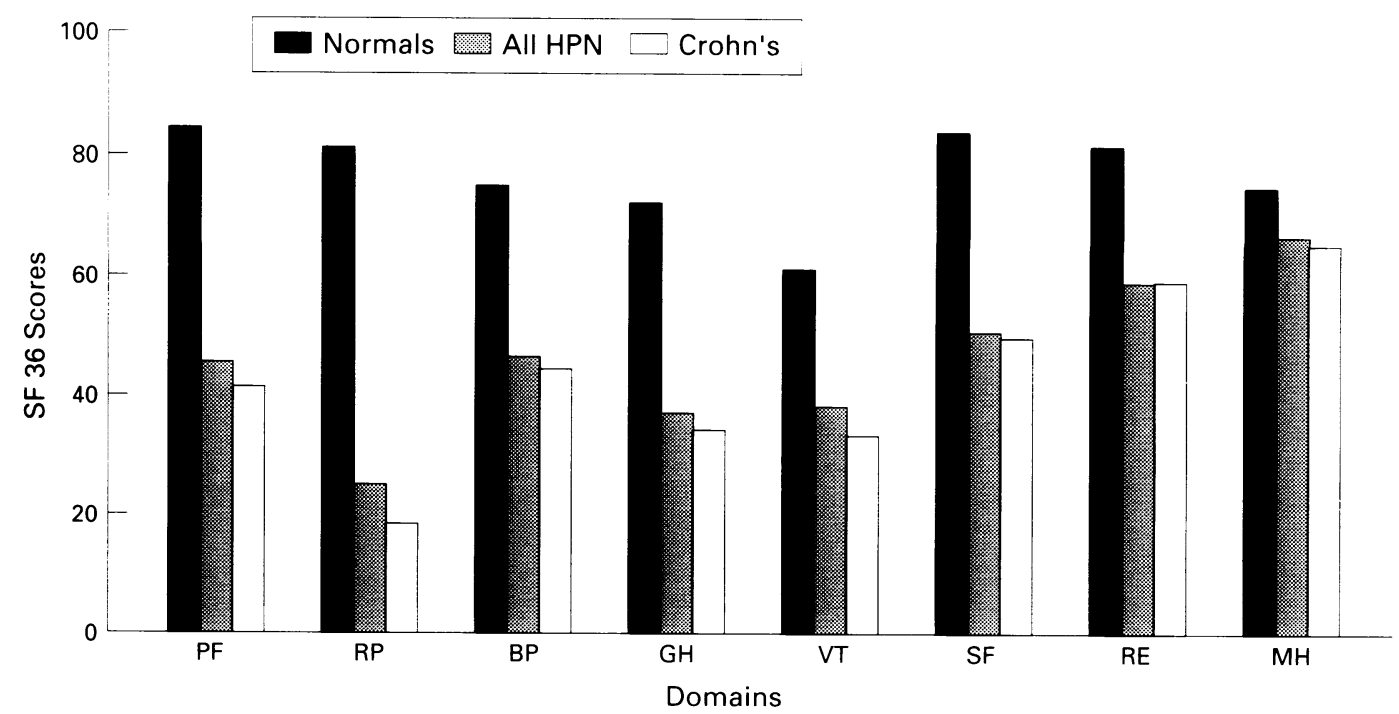

Figure 3: SF 36 scores for the Crohn's disease and other HPN subgroups compared with normal population scores. 


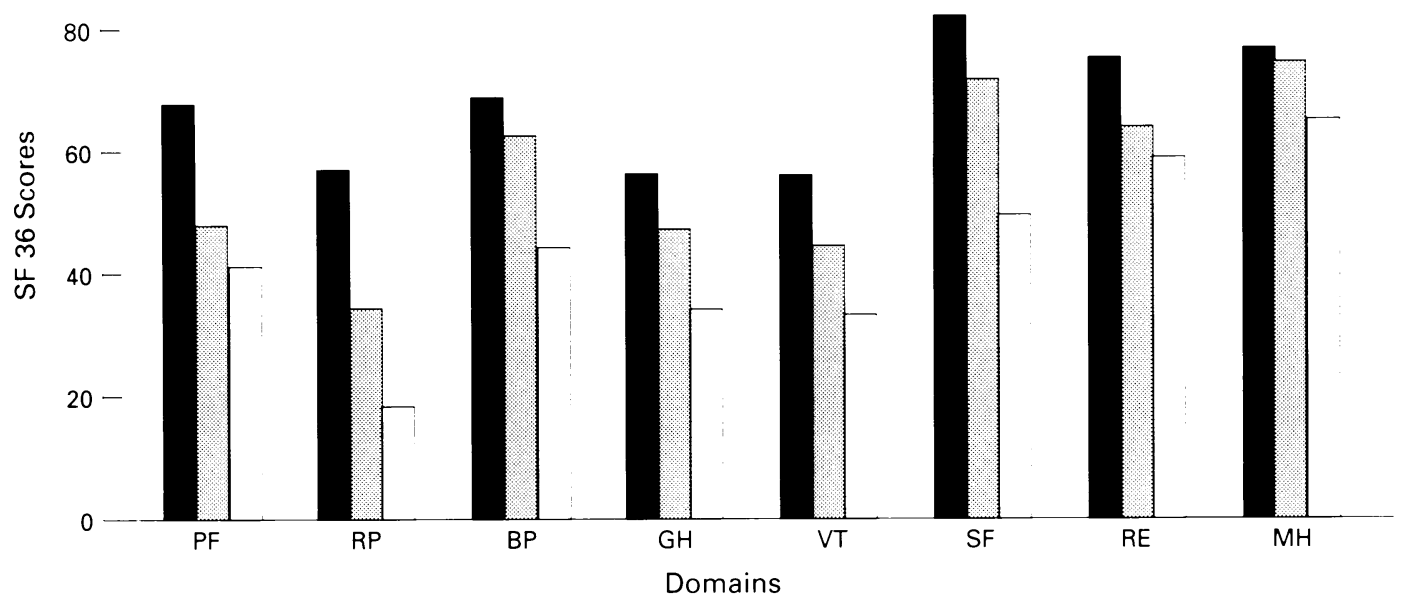

Figure 4: SF 36 scores for patients with Crohn's disease and those with other chronic diseases on HPN.

Price et al ${ }^{11}$ examined early responses to HPN and reported that feelings of anger, anxiety and depression result from the sense of loss sustained by the patient. The loss includes the inability to eat normally, loss of independence, loss of social and work status, and loss of control of bodily functions. Price also reported that the premorbid psychological and physical profile of the patient also has a bearing on the quality of life achieved. The psychological problems that are encountered on HPN tend to disperse as the new way of life becomes accepted ${ }^{8}$ and the end result depends on the level to which their physical health is restored. ${ }^{11}$

Sexual functioning decreases in most patients and only the occasional patient notices an improvement. ${ }^{11}$ Sexual functioning among younger patients was reported as normal; however, it had ceased completely in some of the patients over 55 years of age. ${ }^{9}$ Burnes et al ${ }^{12}$ did not find that older patients had a worse quality of life than the young, but in the cohort studied by O'Hanrahan and Irving, ${ }^{10}$ older patients did less well. Herfindal et al $^{3}$ measured life satisfaction scores of HPN recipients and found them to be in the upper two thirds of the index range; however, these scores were significantly lower than those obtained by patients with end stage renal disease and the general population.

Patients with severe Crohn's disease have usually come to terms with limitations in their lifestyle and often note an improvement in quality of life when started on HPN. This contrasts greatly with those who have a mesenteric vessel occlusion. Often well before their illness, they find their new circumstances quite devastating. ${ }^{389}$

The return to employment is often made on a part time basis as many patients find that they are unable to cope with their previous workload. ${ }^{9}{ }^{11}$ Ladefoged $^{9}$ reported that none of the patients felt able to return to work full time for a variety of reasons including, tiredness, pain, diarrhoea, frequent admission to hospital, and time consuming HPN therapy.
Social adaptation was reported to be best in the middle aged group, who usually have the support of a spouse. ${ }^{13}$

Small bowel transplantation increasingly looks promising, but there are still major problems delaying widespread adoption of the operation. ${ }^{1+4}$ At the present time young well adjusted patients on HPN are being advised to continue HPN until the outcomes of small bowel transplantation improve. It is interesting to note from the study of Carlson et al, ${ }^{15}$ that it was the young patients (with the best quality of life scores) who were most keen on a small bowel transplantation, indicating an unwillingness to accept long term HPN.

The Health Technology Assessment Programme in the United Kingdom is currently examining the evidence of effectiveness of many medical interventions. As part of that assessment it is important to collect data on quality of life as well as other clinical and economic outcomes. A systematic review of the risks and benefits of HPN has located minimal additional data on quality of life outcomes on HPN. ${ }^{16}$ This study has attempted to improve and update the existing evidence.

The results indicate that the patients have, on average, a fairly good quality of life. On the basis that the alternative of not treating these patients is usually death, then the treatment option would seem to be acceptable. This is particularly the case with younger patients, who can usually be restored to a near normal quality of life.

The older patients in our study had significantly lower quality of life scores compared with their younger counterparts. It is anticipated that our aging population of patients on $\mathrm{HPN}$ will pose a future management problem as they become less able to manage the complex aseptic procedure. We have evidence that this age group has a higher incidence of catheter infections ${ }^{17}$ combined with a lower quality of life.

Patients with Crohn's disease receiving HPN had scores that were no different from 
the other disease subgroups, but small numbers in the subgroups prevented statistical confirmation of this fact.

We have a fairly high proportion of patients addicted to opiate analgesics $(16 \%)$ and scores from five of those who responded were very low. Clinical outcomes for this subgroup are also poor. ${ }^{18}$

The percentage of recipients of HPN who were employed at the time of this survey (5\%) was much lower than previously reported $(52 \%)$ in a similar cohort of patients. ${ }^{13}$ The reason for this is unclear but it may be a result of illness related financial benefits.

These results are for a cohort of patients with benign intestinal failure. We do not have any patients with AIDS or active cancer and therefore our results cannot be extended to these groups. The main aim of using HPN for terminal malignant disease must be to improve the quality of life, and to get the patient home. The evidence of efficacy of HPN for these patients is almost non-existent at present and in particular no quality of life data for these patients could be located. This, combined with the dismal survival statistics ${ }^{16}$ indicates an urgent need for an assessment of effectiveness and a comparison with alternative therapeutic strategies.

The technology of HPN is lifesaving for most patients with benign intestinal failure and therefore some reduction in the quality of life is acceptable. When patients are being considered for HPN it is important to judge each case individually. Some consideration should be given to the outcomes that have been shown in the older age group, but this should not be a limiting factor if other aspects of the case are favourable. At present HPN remains the only long term option for the treatment of benign intestinal failure.
We thank Professor A Williams, Paul Kind, Claire Gudex, and Karen Bloor from the Centre for Health Economics, University of York, for advice on quality of life assessment and Jon Deeks from the Centre for Statistics in Medicine, Oxford, for statistical dvice.

This research was funded through the University of Manchester Department of Surgery at Hope Hospital, Salford.

1 Irving MH. Ethical problems associated with the treatment of intestinal failure. Aust $N Z \mathcal{Y}$ Surg 1986; 56: 425-7.

2 Detsky A, McLaughlin JR, Abrams HB, L'Abbe KA Whitwell J, Bombardier C, et al. Quality of life of patients on long term total parenteral nutrition at home. $\mathcal{F} \mathrm{Gen}$ Intern Med 1986; 1: 26-33.

3 Herfindal ET, Bernstein LR, Kudzia K, Wong A. Survey of home nutritional support patients. $f$ Paren Entr Nutr 1989; 13: 255-61

4 Shaffer JL. HPN in Europe - the UK experience [abstract]. Birmingham: European Society of Parenteral and Enteral Nutrition, 1994.

5 EuroQol Group. EuroQol-a new facility for the measurement of health related quality of life. Health Policy 1990 16: 199-208.

6 Medical Outcomes Trust. How to score the SF health survey. Boston: Medical Outcomes Trust. 1994.

7 Ware J. SF 36 health survey, manual and interpretation guide. oston: Medical Outcom, Trust. 1993.

8 Robinovitch AE. HPN: a psycho-social viewpoint. $f$ Paren Entr Nutr 1981; 5: 522-5.

9 Ladefoged K. Quality of life in patients on home parenteral nutrition. $\mathcal{f}$ Paren Entr Nut 1981; 5: 132-7.

10 O'Hanrahan T, Irving MH. The role of HPN in the management of intestinal failure; report of 400 cases. Clinical Nutrition 1992; 11: 331-6.

11 Price BS, Levine EL. Permanent TPN, psychological and social responses to the early stages. F Paren Entr Nutr 1979; 3: 49-52.

12 Burnes JU, O'Keefe SJD, Fleming CR. Home parenteral nutrition: a three year analysis of clinical and laboratory montritoring. $\mathcal{F}$ Paren Entr Nutr 1992; 16: 327-32.

13 Messing B, Landais P, Goldfarb B, Irving MH. Home parenteral nutrition in adults: a multicentre survey in parenteral nutrition in adults: a multice

14 Thompson JS, Langnas AN, Pinch LW, Kaufman S Quigley EMM, Vanderhoof JJ. Surgical approach to the short bowel syndrome. Experience in a population of 160 patients. Ann Surg 1995; 222: 600-7.

15 Carlson GL, Maguire G, Williams N, Bradley A, Schaffer J Irving MH. Quality of life on home parenteral nutrition and attitudes towards intestinal transplantation. A single centre study of 37 patients. Clinical Nutrition (in press).

16 Richards DM, Deeks JI, Shaffer JL Sheldon TA. A systematic review of home parenteral nutrition. London: systematic review of home parenter

17 Williams N, Carlson GL, Scott NA, Irving MH. Incidence and management of catheter related sepsis in patients receiving home parenteral nutrition. Br f Surg 1994; 81: 392-4.

18 Richards DM, Shaffer JL, Scott NA, Irving MH. Opiate and sedative dependence is associated with a poor outcome on HPN. Gut 1996; 38: A41. 